\title{
Confirmation of the Luminous Blue Variable nature of AFGL 2298
}

\author{
J. S. Clark ${ }^{1}$, V. M. Larionov ${ }^{2,3}$, P. A. Crowther ${ }^{1}$, M. P. Egan ${ }^{4}$, and A. Arkharov ${ }^{5}$ \\ 1 Department of Physics and Astronomy, University College London, Gower Street, London, WC1E 6BT, England, UK \\ 2 Astronomical Institute of St. Petersburg University, Petrodvorets, Universitetsky pr. 28, 198504 St. Petersburg, Russia \\ ${ }^{3}$ Isaac Newton Institute of Chile, St. Petersburg Branch \\ ${ }^{4}$ US Air Force Research Laboratory, Space Vehicles Directorate, 29 Randolph Road, Hanscom AFB, MA 01731-3010, USA \\ 5 Central Astronomical Observatory, 196140 St. Petersburg, Russia
}

Received 22 January 2003 / Accepted 13 March 2003

\begin{abstract}
We present new photometric and spectroscopic observations of the stellar source AFGL 2298 (= IRAS 18576+0341) that has recently been proposed as a candidate Luminous Blue Variable (LBV). Our data confirm that the star is a highly luminous B supergiant which is both spectroscopically and photometrically variable. Assuming a distance of $10 \mathrm{kpc}$, comparison of the 2001 June data to synthetic spectra suggest stellar parameters of $T=12.5 \mathrm{kK}, \log \left(L / L_{\odot}\right)=6.2$ and $\dot{M}=5 \times 10^{-5} M_{\odot} \mathrm{yr}^{-1}$. Data obtained in 2002 August indicate an increase in both temperature $(=15 \mathrm{kK})$ and mass loss rate $\left(=1.2 \times 10^{-4} M_{\odot} \mathrm{yr}^{-1}\right)$ at constant bolometric luminosity. These physical parameters place AFGL 2298 at the Humphreys-Davidson limit for the most luminous stars known. We conclude that the position of AFG 2298 in the HR diagram, the significant variability observed between 1999-2002 and presence of a massive ejection nebula are consistent with a classification of AFGL 2298 as a bona fide LBV.
\end{abstract}

Key words. stars: emission-line, Be - stars: circumstellar matter

\section{Introduction}

As massive, luminous stars evolve from the Main Sequence to become hydrogen depleted Wolf Rayet stars they shed large quanties of matter over a very short period of time. The period of enhanced mass loss is associated with the Luminous Blue Variable (LBV) phase, which is characterised - and indeed defined - by the presence of spectroscopic and photometric variability on timescales from months to years and which in some cases - the so called "giant eruptions" - leads to an increase in both the bolometric luminosity and mass loss rate of the star.

Given their rarity, it is expected that the LBV phase is very short, possibly only of the order of a few $\times 10^{4} \mathrm{yr}$ (Voors et al. 2000a). However, the lack of an accurate galactic census prevents a refinement of this estimate and consequently much effort has been applied to the identification of new LBV candidates (cLBV). Recently, an increasing number of highly obscured cLBVs have been identified on the basis of near-IR spectroscopy (e.g. the Pistol Star; Figer et al. 1998) or mid-IR - radio imaging of the circumstellar ejection nebulae that characterise many of the known LBVs (e.g. Wra 17-96; Egan et al. 2002).

One recently identified cLBV is the highly reddened source AFGL 2298 (= IRAS 18576+0341; we adopt the AFGL designation for brevity). Ueta et al. (2001; U01) detected

Send offprint requests to: J. S. Clark, e-mail: jsc@star.ucl.ac.uk a compact, bipolar nebula around a central stellar source from mid-IR (10-20 $\mu \mathrm{m})$ imaging, which they further identify as a strong radio source ${ }^{1}$. Interpreting the mid-IR emission as arising from an evolved star surrounded by a thick dusty torus inclined to the line-of-sight, U01 employed a radiative transfer code to determine the properties of both star and nebula. They found that the nebular emission is dominated by cool (115-135 K) dust with a possible mixed (O \& C rich) chemistry, surrounding a highly luminous $\left(10^{6.4} L_{\odot}\right)$ early type star $\left(T_{\text {eff }}=15 \pm 6 \mathrm{kK}\right)$, seen at a large distance $(10 \pm 3 \mathrm{kpc})$.

U01 also presented 4 epochs of photometry which appears to indicate the presence of significant variability and hence proposed that AFGL 2298 was a strong cLBV. Subesquently, Pasquali \& Comorón (2002; PC02) presented a low resolution $(R \sim 240)$ HK spectrum of the central source which revealed the presence of strong $\mathrm{H}$ I and He I emission, as might be expected from a hot luminous star with a high mass loss rate. On the basis of this spectrum PC02 concluded that AFGL 2298 is a bona fide LBV, which they associate with the hot Ofpe/WN9

${ }^{1}$ U01 claim a positional coincidence with the radio source GPSR5 $37.278-0.226$ and quote fluxes of $S_{5 \mathrm{GHz}}=78.1 \mathrm{mJy}$ (Becker et al. 1994 ) and $S_{1.4 \mathrm{GHz}}=8.1 \pm 0.7 \mathrm{mJy}$ (NVSS survey; Condon et al. 1999). However, these fluxes yield a spectral index greater than expected for optically thick emission and examination of the fields suggests that background source confusion renders the values quoted as unreliable; indeed Becker et al. (1994) quote $S_{1.4 \mathrm{GHz}}=198 \mathrm{mJy}$ for the same source. 


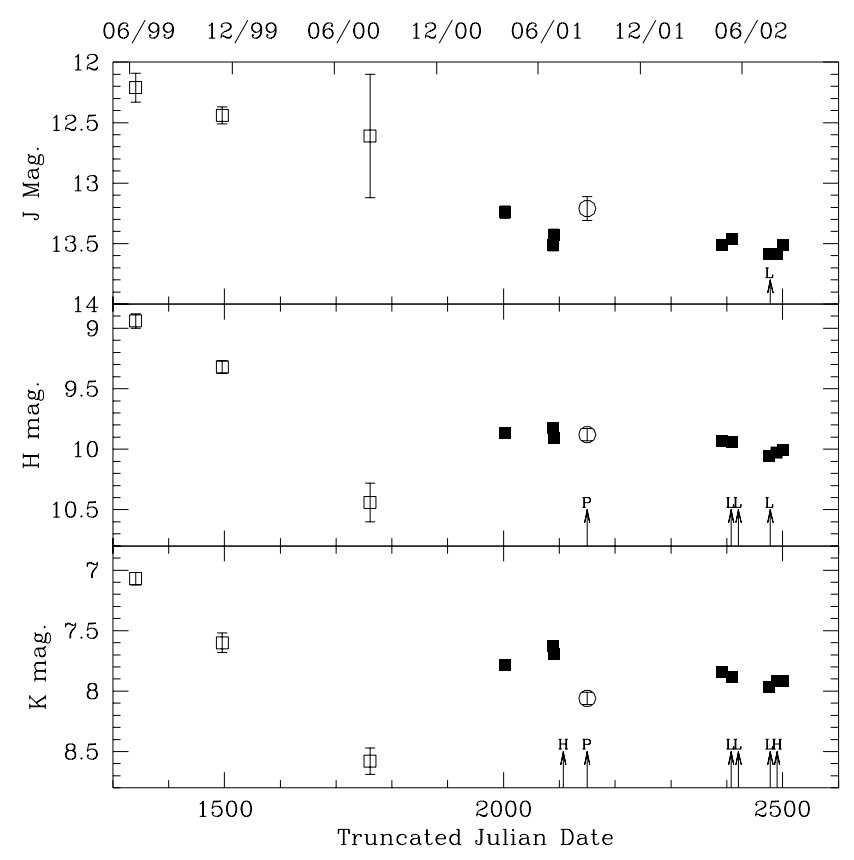

Fig. 1. JHK broadband photometry of AFGL 2298. Open squares represent the data of U01 and circles those of PC02. Errorbars are indicated; where none are visible they are smaller than the symbol size. The date spectra were taken is indicated by an arrow and the resolution by either Low or High. The date of the spectrum presented by PC02 is indicated by a $\mathbf{P}$.

phase of the LBV variability cycle. In this paper we report on new, multi-epoch spectroscopic and photometric observations of AFG 2298 which we use to clarify both the nature of the proposed variability and the physical parameters of the central star.

\section{Observations}

\subsection{Photometry}

Near-IR JHK broadband photometric observations of AFGL 2298 were obtained at the AZT-24 $1.1 \mathrm{~m}$ telescope in Campo Imperatore (Italy). The SWIRCAM $256 \times 256 \mathrm{HgCdTe}$ detector was employed, yielding a scale of $1.04 \mathrm{arcsec} / \mathrm{pix}$, resulting in a $\sim 4^{\prime} \times 4^{\prime}$ field of view. Standard techniques of sky subtraction and flatfielding were applied and the results are presented alongside previously published data in Fig. 1 and Table 1.

On the basis of their photometric observations both U01 and PC02 conclude that AFGL 2298 is variable $(\Delta J, H$ and $K \sim 0.5$ mag.) on timescales from months-years. Our new data extends the baseline of observations and allows us to investigate these claims. Taken in isolation, our observations suggest that AFGL 2298 is indeed variable, with at least one pair of observations in each waveband differing from each other at the $\geq 5 \sigma$ level. Note that the magnitude of variability in our data is less that that reported in U01 $\left(\Delta J_{\max } \sim 0.3 \mathrm{mag}\right.$, $\Delta H_{\max } \sim 0.2 \mathrm{mag}$ and $\left.\Delta K_{\max } \sim 0.3 \mathrm{mag}\right)$. Comparison to the previously published data of U01 also demonstrates variability at the $\geq 5 \sigma$ level, but suggest that, if real, the rate of the long term fading U01 infer from their data has substantially
Table 1. Broad band $J H K$ photometry for AFGL 2298; the data are from ${ }^{1}$ Garcia-Lario et al. (1997), ${ }^{2}$ Ueta et al. (2001) and ${ }^{3}$ Pasquali \& Comeron (2002).

\begin{tabular}{lccc}
\hline \hline Date & $J$ & $H$ & $K$ \\
\hline 1989 May-June $^{1}$ & $12.00 \pm 0.30$ & $8.99 \pm 0.03$ & $6.91 \pm 0.02$ \\
1999 Jun. 12 & $12.21 \pm 0.12$ & $8.94 \pm 0.06$ & $7.07 \pm 0.05$ \\
1999 Nov. 14 $^{2}$ & $12.44 \pm 0.07$ & $9.32 \pm 0.05$ & $7.60 \pm 0.08$ \\
2000 Aug. 5 & $12.61 \pm 0.51$ & $10.44 \pm 0.16$ & $8.58 \pm 0.11$ \\
2001 Apr. 4 & $13.24 \pm 0.05$ & $9.87 \pm 0.03$ & $7.78 \pm 0.02$ \\
2001 Jun. 29 & $13.51 \pm 0.05$ & $9.82 \pm 0.03$ & $7.63 \pm 0.02$ \\
2001 Jun. 30 & $13.43 \pm 0.05$ & $9.91 \pm 0.03$ & $7.69 \pm 0.02$ \\
2001 Aug. 29 & $13.21 \pm 0.1$ & $9.88 \pm 0.05$ & $8.06 \pm 0.05$ \\
2002 Apr. 27 & $13.51 \pm 0.02$ & $9.93 \pm 0.02$ & $7.84 \pm 0.02$ \\
2002 May 14 & $13.46 \pm 0.02$ & $9.94 \pm 0.02$ & $7.88 \pm 0.02$ \\
2002 Jul. 18 & $13.59 \pm 0.02$ & $10.05 \pm 0.02$ & $7.97 \pm 0.02$ \\
2002 Aug. 1 & $13.58 \pm 0.02$ & $10.03 \pm 0.02$ & $7.92 \pm 0.02$ \\
2002 Aug. 14 & $13.51 \pm 0.02$ & $10.01 \pm 0.02$ & $7.91 \pm 0.02$ \\
\hline
\end{tabular}

decreased since late 2000. We further note that in comparison to our data the minimum in the lightcurves during mid-2000 identified in PC02 is only significant at the $\geq 5 \sigma$ level in the $K$ band lightcurve.

\subsection{Spectroscopy}

Near-IR spectroscopic observations of AFGL 2298 were made in service time with the United Kingdom Infrared Telescope (UKIRT) on 2001 June 17 and 2002 August 6, using the Cooled Grating Spectrometer (CGS4). Observations between 2.0-2.5 microns were made using the long focal length camera plus the 40 line/mm grating, resulting in a resolving power of $800.2 \times$ oversampling of the spectrum was employed to correct for bad pixels in the array. Standard throws along the slit to maximise the $\mathrm{S} / \mathrm{N}$ were employed.

Initial data reduction was carried out at the telescope using the ORACDR software package. This removes bad pixels, debiases, flat-fields, linearity corrects and interleaves oversampled scan positions. The subsequent stages of data reduction, comprising of sky subtraction, extraction, derippling and wavelength calibration using observations of a CuAr lamp, were carried out using the Starlink-supported package FIGARO. Division by main sequence A \& G star telluric standards was accomplished following the procedure described in Egan et al. (2002). Measurement of the centroids of the spectral features and measurement of the equivalent widths $(E W)$ of the lines was accomplised with DIPSO; these are presented in Table 2.

These spectra were supplemented by lower resolution $(R \sim 270)$ observations made on 2002 May 13, May 26 and July 30 from the AZT-24 $1.1 \mathrm{~m}$ telescope, with the IR imaging camera SWICAM and the IH and HK band grisms. These provided spectral coverage between $0.84-1.32 \mu \mathrm{m}$ and $1.45-2.38 \mu \mathrm{m}$ respectively. No variability was observed between any of these observations - hence we present the mean of these spectra in Fig. 3, and EWs in Tables 2 and 3.

Our spectra confirm results of $\mathrm{PC} 02$ who find a spectrum dominated by $\mathrm{HI}, \mathrm{He}$ I and low excitation metallic (Fe II and $\mathrm{Mg}$ II) emission. Weak $\mathrm{Na}$ I emission is also apparent in 


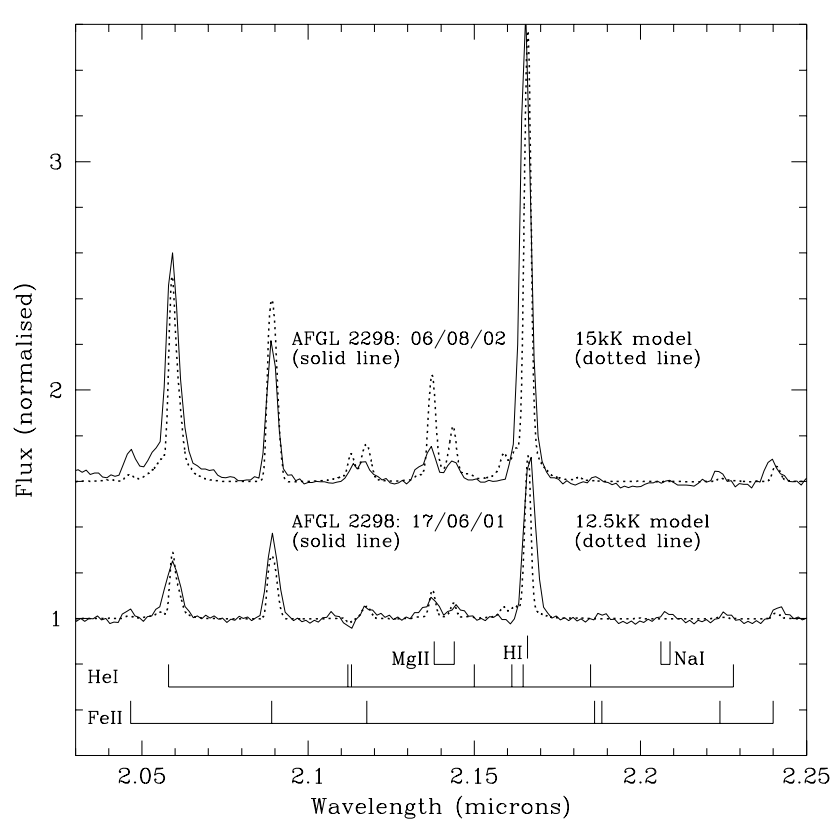

Fig. 2. $K$ band spectra of AFGL 2298 obtained in 2001 June 17 and 2002 August 6 with the CGS 4 at UKIRT (solid lines) overplotted with the synthetic spectra described in Sect. 3. Prominent transitions are indicated.

the CGS4 spectrum obtained on 2001 June 17. Significant variability is observed, with an increase in line strength by a factor of $\sim 3$ for the He I $2.058 \mu \mathrm{m}$ and $\mathrm{Br} \gamma$ lines between 2001-2002, along with a similar but less dramatic increase in the strength of the low excitation metallic transitions. Of particular interest is the transition between absorption and emission in He I $2.112 / 3 \mu \mathrm{m}$ and the change in the He I $2.058 \mu \mathrm{m}$ : Fe II $2.089 \mu \mathrm{m}$ ratio ( $\leq 1$ in 2001 June-August $\rightarrow>1$ between 2002 May-August). Comparison to the photometric lightcurve indicates that AFGL 2298 was also photometrically variable during this period, although the sparse nature of the dataset precludes confirmation of any coherent trends in brightness.

\section{Discussion}

Comparison of our new spectra to those of other massive evolved stars (e.g the LBV and $\mathrm{sgB}[\mathrm{e}]$ spectra presented by Morris et al. 1996) reinforces the conjecture of U01 and PC02 that AFGL 2298 is likely to be a luminous $B$ supergiant. In particular we note a remarkable similarity to the spectra of the cLBVs G79.29+0.46 (Voors et al. 2000b), the Pistol Star (Figer et al. 1998) and Wra 17-96 (Egan et al. 2002).

In order to determine the stellar parameters of AFGL 2298 we have computed a series of $B$ supergiant models using the Hillier \& Miller (1998) line blanketed, non-LTE model atmospheric code, for comparison with our near-IR spectra. Model calculations were carried out using similar atomic datasets and elements to that undertaken by Drissen et al. (2001) for NGC 2363-V1. Unfortunately, given the narrow wavelength range and intermediate resolution of the CGS4 observations, we are forced to adopt a terminal wind velocity $\left(200 \mathrm{~km} \mathrm{~s}^{-1}\right)$ and $\mathrm{H} / \mathrm{He}$ abundance ( 5 by number) typical of Luminous Blue

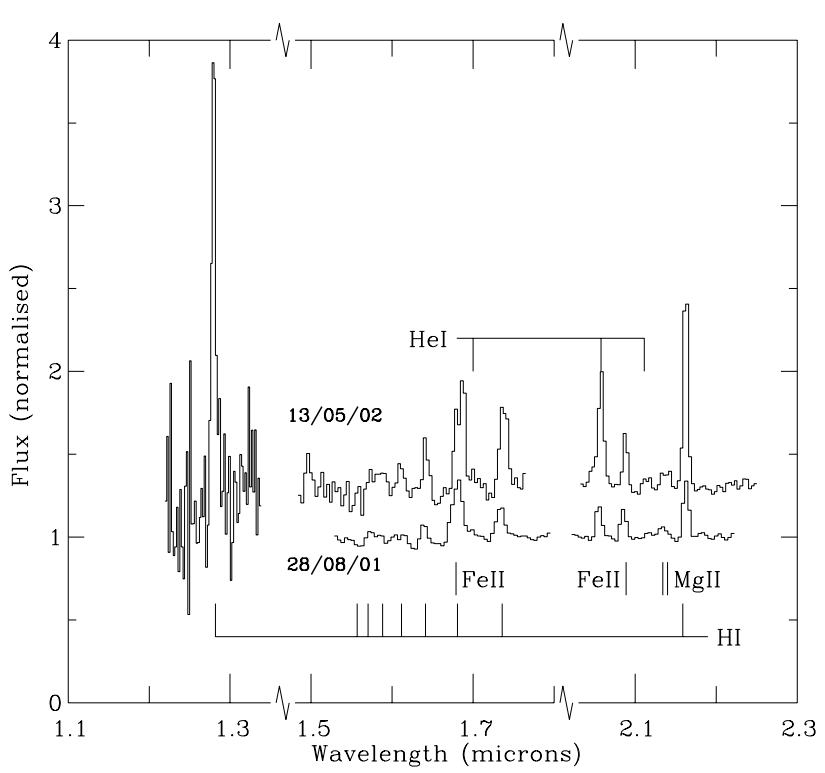

Fig. 3. Low resolution $I J \& H K$ band spectra of AFGL 2298 from 28 August 2001 (PC02) and 2002 May 13 (Campo Imperatore).

Table 2. Line identifications and equivalent widths ( $E W \mathrm{~s}$; given in $\AA$ ) for the $K$ band $(\sim 2-2.35 \mu \mathrm{m})$ spectra of AFGL 2298. Note that we have adopted the convention that emission lines are quoted as positive throughout the table. We estimate errors of \pm 10 per cent for the UKIRT spectra and \pm 20 per cent for the spectra from Campo Imperatore. Dates are given in month/year form for brevity; the exact dates of spectra are listed in Sect. 2.2. Note that within the observational uncertainties the $E W$ measured from the 3 low resolution spectra taken between 2002 May-July are identical to one another (hence we only quote a mean value) while the values obtained from 2001 August (PC02) are identical to those obtained in 2001 June (and are also omitted for brevity).

\begin{tabular}{|c|c|c|c|}
\hline $\begin{array}{l}\text { Date } \\
\text { Transition }\end{array}$ & $06 / 01$ & $\begin{array}{c}05 / 02 \\
E W(\AA)\end{array}$ & $08 / 02$ \\
\hline$[\mathrm{Fe}$ II $]\left(\mathrm{a}^{4}{ }_{5 / 2}-\mathrm{a}^{2} \mathrm{P}_{3 / 2}\right)$ & 1.9 & $?$ & 5.0 \\
\hline He I $\left(2 s^{1} S-2 p^{1} P^{0}\right) 2.058$ & 14.3 & 58 & 49.6 \\
\hline $\mathrm{Fe}$ II $\left(\mathrm{z}^{4} \mathrm{~F}_{3 / 2}-\mathrm{c}^{4} \mathrm{~F}_{3 / 2}\right) 2.091$ & 15.8 & 24 & 24.2 \\
\hline He I $\left(3 p^{3} \mathrm{P}^{0}-4 s^{3} \mathrm{~S}\right) 2.112$ & -0.8 & 8 & 6.3 \\
\hline$+\operatorname{He} I\left(3 p^{1} P^{0}-4 s^{1} S\right) 2.113$ & bl & bl & bl \\
\hline Fe II $\left(c^{4} \mathrm{P}_{3 / 2}^{0}-\mathrm{z}^{4} \mathrm{D}^{0}{ }_{5 / 2}\right) 2.1177$ & 2.1 & $?$ & bl \\
\hline $\operatorname{Mg}$ II $\left(5 s^{2} \mathrm{~S}_{1 / 2}-5 \mathrm{p}^{2} \mathrm{P}^{0}{ }_{1 / 2}\right) 2.138$ & 5.2 & 13 & 6.9 \\
\hline $\operatorname{Mg}$ II $\left(5 \mathrm{~s}^{2} \mathrm{~S}_{1 / 2}-5 \mathrm{p}^{2} \mathrm{P}^{0}{ }_{1 / 2}\right) 2.144$ & 2.8 & bl & 4.7 \\
\hline He I $\left(4 p^{3} P-7 s^{3} S\right) 2.15$ & -0.2 & $?$ & - \\
\hline $\operatorname{Br} \gamma 2.1661$ & 31.0 & 90 & 81.6 \\
\hline He I $\left(4 p^{1} P-7 d^{1} D\right) 2.185$ & 1.5 & - & 1.5 \\
\hline $\mathrm{NaI}\left(4 \mathrm{p}^{2} P_{3 / 2}^{0}-4 \mathrm{~s}^{2} \mathrm{~S}_{1 / 2}\right) 2.209$ & 2.4 & - & - \\
\hline$+\mathrm{NaI}\left(4 \mathrm{p}^{2} P_{3 / 2}^{0}-4 \mathrm{~s}^{2} \mathrm{~S}_{1 / 2}\right) 2.206$ & bl & - & - \\
\hline Fe II $\left(\mathrm{a}^{2} \mathrm{H}_{11 / 2}-\mathrm{a}^{2} \mathrm{G}_{9 / 2}\right) 2.224$ & 1.2 & - & 2.2 \\
\hline Fe II $\left(a^{4} P_{1 / 2}-a^{2} P_{3 / 2}\right) 2.244$ & 2.6 & - & 5.7 \\
\hline
\end{tabular}

Variables during mid- $B$ evolutionary phases (Crowther 1997). All other elements are set at solar values.

For the purpose of our modelling, we adopt $d=10 \mathrm{kpc}$ and $E(B-V)=9$ mag (U01), noting that these exceed those 
Table 3. Line identification and equivalent widths (given in $\AA$ ) between $\sim 1.1-1.8 \mu \mathrm{m}$ for AFGL 2298 between 2001 August (PC02) and 2002 May. We estimate errors to be \pm 20 per cent of the quoted $E W$. Note that within the observational uncertainty no variability was apparent over the 3.5 month period between 2002 May-July.

\begin{tabular}{lcc}
\hline \hline $\begin{array}{l}\text { Date } \\
\text { Transition }\end{array}$ & $08 / 01$ & $05 / 02$ \\
\hline $\mathrm{Pa} \beta$ & - & 250 \\
$\mathrm{Br} 13$ & - & 20 \\
$\mathrm{Br} 12$ & 10 & 31 \\
$\mathrm{Br} 11+$ & 50 & 120 \\
$\mathrm{Fe} I \mathrm{II}\left(\mathrm{z}^{4} \mathrm{~F}_{9}-\mathrm{c}^{4} \mathrm{~F}_{7}\right) 1.6787$ & $\mathrm{bl}$ & $\mathrm{bl}$ \\
$\mathrm{Br} 10$ & 25 & 80 \\
\hline
\end{tabular}

suggested by PC02, and thus provide an upper limit to the luminosity and mass-loss rate of AFGL 2298. For this assumed reddening, the near-IR spectral energy distribution (which includes contributions from both star and stellar wind) is in good agreement with the de-reddened $J H K$ photometry, such that there is no evidence for hot dust in AFGL 2298.

The stellar parameters of AFGL 2298 are estimated for the two available epochs by simultaneously reproducing the $\operatorname{Br} \gamma$, $\mathrm{He}$ I+ Fe II $2.058 \mu \mathrm{m}$ and Fe II $2.09 \mu \mathrm{m}$ emission profiles, together with the H-band photometry. Note that charge exchange, important for many Fe II transitions, is accounted for following Hillier et al. (2001). Line blanketing is treated using a global Doppler line width of $10 \mathrm{~km} \mathrm{~s}^{-1}$, whilst wind clumping is neglected.

Despite the limited diagnostics, the best fit parameters and predicted broadband photometric magnitudes resulting from this modeling are presented in Table 4 and the synthetic spectra overplotted in Fig. 2. The modeling confirms that AFGL 2298 is a highly luminous $\left(10^{6.1} L_{\odot}\right)$ mid- $B\left(T_{\text {eff }}=13.5-15 \mathrm{kK}\right)$ supergiant with parameters consistent with those predicted by U01 on the basis of radiative modeling of the dusty nebula. This range of temperatures indicates that AFGL 2298 occupies the region of the HR diagram populated by other LBVs (which have temperatures ranging from $\sim 8 \mathrm{kK}$ to $\sim 20 \mathrm{kK}$; Crowther 1997) ${ }^{2}$. Indeed the temperature variations observed indicate that AFGL 2298 actually moved from above to below the empircal Humphreys-Davidson (H-D) limit for massive stars (Humphreys \& Davidson 1979) between 2001-2002.

We note that even assuming that the distance is in error by a factor of 2 still results in stellar parameters for AFGL 2298 that are fully consistent with an LBV identification (e.g. $\log \left(L_{*} / L_{\odot}\right)=5.6$ and $\dot{M}=10^{-4.7} M_{\odot} \mathrm{yr}^{-1}$ by adopting the scaling factors for the spectroscopically similar star HD 316285; Hillier et al. 1998). An alternative identification of AFGL 2298 as a post-AGB object (e.g. Garcia-Lario et al. 1997) appears untenable, given that in order to force the luminosity and mass loss rate of AFGL 2298 down to values

\footnotetext{
${ }^{2}$ We note that PC02 suggest a higher temperature $(>20 \mathrm{kK})$ for AFGL 2298, based on the strength of the He I $2.058 \mu$ m transition in their low resolution spectrum. However the presence of low excitation species in our spectrum (Fe II, Mg II and Na I) - absent for higher temperature Ofpe/WN9 objects (Bohannan \& Crowther 1999) - demonstrates that AFGL 2298 has a lower temperature than this estimate.
}

Table 4. Stellar parameters and predicted broadband photometry of AFGL 2298 in 2001 June and 2002 August, derived from comparison to NLTE synthetic spectra (see Sect. 3 for details). Note that both $v_{\exp }$ and the $\mathrm{H} / \mathrm{He}$ abundance ratio cannot be determined directly from the present dataset and hence were set to values appropiate to high luminosity mid- $B$ supergiants; note that solar Fe $\&$ Mg abundances are assumed.

\begin{tabular}{lcc}
\hline \hline Parameter & 2001 & 2002 \\
\hline$v_{\text {exp }}\left(\mathrm{km} \mathrm{s}^{-1}\right)$ & 200 & 200 \\
$\mathrm{H} / \mathrm{He}$ & 5 & 5 \\
$T_{\text {eff }}(\mathrm{kK})$ & 12.5 & 15 \\
$\log \left(L_{*} / L_{\odot}\right)$ & 6.2 & 6.1 \\
$R_{*}\left(R_{\odot}\right)$ & 260 & 165 \\
$\log (\dot{M})\left(M_{\odot} \mathrm{yr}^{-1}\right)$ & -4.3 & -3.9 \\
\hline Photometry & & \\
\hline$R$ & 25.4 & 25.8 \\
$I$ & 18.9 & 19.3 \\
$J$ & 13.4 & 13.7 \\
$H$ & 9.8 & 10.0 \\
$K$ & 7.9 & 7.9 \\
$L$ & 6.1 & 5.9 \\
\hline
\end{tabular}

commonly expected for such objects, an extremely small distance $(\leq 600 \mathrm{pc})$ for the observed reddening is required. Furthermore, the observed spectrum is not consistent with such a suggestion, lacking emission from forbidden lines of both low and high excitation (e.g. Lamers et al. 1998).

Therefore, we conclude that the position of AFG 2298 on the HR diagram, the presence of a massive $\left(\sim 10 M_{\odot}\right)$ dusty ejection nebulae and pronounced spectral and photometric variability observed (Sect. 2) provides a compelling argument for the identification of AFGL 2298 as a bona fide LBV as suggested by U01 and PC02.

Taken at face value, the constant bolometric luminosity of AFGL 2298 between 2001-2002 suggest that it is at present undergoing one of the quasi periodic excursions across the HR diagram that characterise LBVs. However, consideration of the long term behaviour of NGC 2363-V1 (Drissen et al. 2001) suggests that plateau states of moderate variability at $\sim$ constant bolometric luminosity can also occur during the so called "giant outbursts" of LBVs; hence we are unable to conclude that AFGL 2298 is not in a similar state at present. One result of this uncertainty is that we are unable to state whether the luminosity determined from the modeling represents the "quiescent" luminosity of AFGL 2298. Thus we are also prevented from inferring a progenitor mass for AFGL 2298 by comparison to the theoretical evolutionary tracks for massive stars available in the literature; however we note that under the assumption that AFGL 2298 is undergoing a "normal" LBV excursion accross the HR diagram the progenitor mass is likely to be in excess of $60 M_{\odot}$.

While AFGL 2298 appears to have shown significant photometric variability between 1999-2001 the spectroscopic changes reported in this paper have occured in an 8 month period between 2001 August \& 2002 May. For a constant bolometric luminosity between 2001-2002 the increase in $T_{\text {eff }}$ 
corresponds to a change in stellar radius from $\sim 205 R_{\odot}-$ $\sim 166 R_{\odot}$. This implies that the stellar surface contracted at average rate of $-2.3 \mathrm{~km} \mathrm{~s}^{-1}$ during this time. Such a value appears directly comparable to the rate of expansion $\left(+4 \mathrm{~km} \mathrm{~s}^{-1}\right)$ and contraction $\left(-1.4 \mathrm{~km} \mathrm{~s}^{-1}\right.$ ) of NGC 2363-V1 (Drissen et al. 2001) and $\sim+2.3 \mathrm{~km} \mathrm{~s}^{-1}$ inferred for AG Car between 1990 December-1991 October (Leitherer et al. 1994).

The change in photospheric radius is also accompanied by an increase of the mass loss rate $(\dot{M})$ by a factor of 2.4 to $1.2 \times 10^{-4} M_{\odot} \mathrm{yr}^{-1}$ by 2002 May. The behaviour of $\dot{M}$ with the changes in $T_{\text {eff }}$ and $R_{*}$ during the constant bolometric luminosity excursions of LBVs has been the subject of much speculation, but quantitative conclusions have been complicated by the small number of observations available, and often seemingly contradictory results ${ }^{3}$. For example $\mathrm{R} 71$ $\left(\dot{M} \propto 1 / T_{\text {eff }} \propto R_{*} ;\right.$ Wolf et al. 1981) and R $110\left(\dot{M} \propto T_{\text {eff }} \propto\right.$ $1 / R_{*}$; Stahl et al. 1990) show diametrically opposing behaviour, while more recent analysis of AG Car (Leitherer et al. 1994) and NGC 2363 V1 (during the 1996-2000 "plateau" phase of the current giant outburst; Drissen et al. 2001) show little or no variability in $\dot{M}$ despite significant changes in $T_{\text {eff }}$ and $R_{*}$. Nevertheless the inverse correlation between $\dot{M}$ and $R_{*}$ inferred for AFGL 2298 support the conclusion of Leitherer et al. (1994) from analysis of AG Car, that the changes in the stellar radius in LBVs are not due to the formation of a pseudophotosphere in a wind with high $\dot{M}$ (e.g. Appenzeller 1986; Davidson 1987) ${ }^{4}$. Indeed the results of Hillier et al. (2001) for $\eta$ Car suggest that a $\dot{M} \geq 10^{-3} M_{\odot} \mathrm{yr}^{-1}$ is required to completely veil the stellar "photosphere".

The $\dot{M}$ of AFGL 2298 is larger than that typically adopted for (lower luminosity) LBVs and "normal" BSGs $\left(\sim 10^{-5} M_{\odot} \mathrm{yr}^{-1}\right.$; e.g. Lamers \& Nugis 2002) and is only exceeded by those of the LBVs $\eta$ Carinae (Hillier et al. 2001), HD 5980, NGC 2363-V1 (Drissen et al. 2001) and the cLBV HD 316285 (Hillier et al. 1998). However, U01 report a total mass for the ejection nebula associated with AFGL 2298 of $10 M_{\odot}$ and kinematic age of $1.2 \times 10^{4} \mathrm{yr}^{5}$. Assuming a constant $\dot{M}$ during the formation of the nebula, this implies that $\dot{M}=8.5 \times 10^{-4} M_{\odot} \mathrm{yr}^{-1}$ at this time, a factor of $\sim 7$ greater still than we infer for 2002. Adopting an expansion velocity of $200 \mathrm{~km} \mathrm{~s}^{-1}$ (equal to that assumed during modeling) during this period results in $\dot{M}=3.4 \times 10^{-3} M_{\odot} \mathrm{yr}^{-1}$, or

\footnotetext{
${ }^{3}$ However note that due to the small number of observations comparisons are being drawn between stars of very different temperature $\&$ luminosity ranges, metallicities and evolutionary stages.

${ }^{4}$ We are forced to make the implicit assumption that $v_{\exp }$ remains constant between the observations. However, given the contraction of AFGL 2298 one might expect an increase in $v_{\exp }$ if the correlation between $v_{\text {escape }}$ and $v_{\text {exp }}$ observed for other OB and LBV stars is maintained (e.g. NGC 2363-V1, HD 5980 and AG Car; Drissen et al. 2001; Leitherer et al. 1994), which would imply an even larger increase in $\dot{M}$ than we derive.

${ }^{5}$ U01 quote several different values of $R_{\text {in }}$ and $R_{\text {out }}$ based on imaging and modeling results; we adopt the inner radius that resulted from modeling, $R_{\text {in }}=0.12 \mathrm{pc}$, and a value of $R_{\text {out }}=6 R_{\text {in }}$ (cf. Sect. 5.3 of U01) noting that this value is poorly constrained but exceeds the outer radius of the observed mid-IR emission. An expansion velocity $v_{\text {exp,neb }}=50 \mathrm{~km} \mathrm{~s}^{-1}$ and a gas: dust ratio of 100 were also used.
}

a factor of $\sim 30$ greater than today. While extreme, such $\dot{M}$ are not unprecedented, with Hillier et al. (1998) finding a present day $\dot{M}=10^{-3} M_{\odot} \mathrm{yr}^{-1}$ for $\eta$ Car. Moreover, we note that similarly massive $\left(\geq 10 M_{\odot}\right)$ dusty envelopes have also been found around AG Car (Voors et al. 2000a) and the cLBVs Wra 17-96 (Egan et al. 2002), the Pistol Star (Figer et al. 1999) and G79.49+0.26 (Higgs et al. 1994), suggesting that such high $\dot{M}$ may be characteristic of some, or all (c)LBV.

Given the similarities in the stellar properties of AFGL 2298 and AG Car (e.g. Leitherer et al. 1994) it is notable that they also share similar nebular properties. As well as an asymmetric, possibly toroidal, distribution of material, both nebulae appear to have comparable sizes, masses and ages and, subject to the accurate determination of $R_{\text {out }}$, the $\dot{M}$ required to form both nebulae are also comparable (U01, Voors et al. 2000a). Comparison of chemical composition and grain size is complicated by the differing assumptions of the two studies. Nevertheless, direct comparison of the mid-IR spectra of both objects reveals strong emission features between $\sim 3-11 \mu \mathrm{m}-$ usually attributed to Polycyclic Aromatic Hydrocarbons - are present while emission features at $\sim 30 \mu \mathrm{m}$ indicate the presence of silicates, suggesting that both nebulae share a mixed $\mathrm{O}+\mathrm{C}$ rich chemistry. Modeling of the spectral energy distributions of both sources suggests that the temperatures of the major (O-rich) dust components are comparable and that an additional population of small warm grains must also be present.

Indeed, the only significant difference between the two sources is that Voors et al. (2000a) propose that the nebula around AG Car contains large $(>1 \mu \mathrm{m})$ grains which appear to be absent from AFGL 2298. However we note that while the modeling of Voors et al. (2000a) invokes a power law distribution of grain sizes (in comparison to the single grain size adopted by U01) the calculations are only completed in 1d, compared to the $2 \mathrm{~d}$ treatment of U01 which can treat scattering correctly. Hence it is possible that a full $2 \mathrm{~d}$ solution for AG Car may obviate the need for very large grains (J. Yates, private communication 2002) and we are currently investigating this possibility. Indeed, with the possible exception of the grain size and the current, variable $\dot{M}$ of AFGL 2298 - which is presently an $\sim$ order of magnitude larger than AG Car (Leitherer et al. 1994; but see Voors et al. 2000a) - both stars and their associated nebulae appear $\sim$ identical to one another.

\section{Conclusions}

We have presented new near-IR spectroscopic and photometric observations of the cLBV AFGL 2298. These observations confirm the results of $\mathrm{U} 01$ and PC02 that the star is photometrically variable and also demonstrate significant spectroscopic variability for the first time. Preliminary modeling of the stellar spectra confirm that AFGL 2298 is a highly luminous $\left(10^{6.2} L_{\odot}\right)$ mid B supergiant. These parameters place AFGL 2298 precisely at the HD limit for massive stars, in the region of the HR diagram occupied by other known LBVs. The combination of stellar parameters, significant spectroscopic and photometric variability and presence of a massive dusty ejection nebula lead to the conclusive identification of AFGL 2298 as a new LBV. 
We find that in an 8 month period between 2001-2002 the temperature of AFGL 2298 increased from $12.5 \mathrm{kK}-15 \mathrm{kK}$. This occured at constant bolometric luminosity, implying a reduction in stellar radius from $260-165 R_{\odot}$, at an average rate of $-2.3 \mathrm{~km} \mathrm{~s}^{-1}$; directly comparable to the rate of change of the radius of NGC 2363-V1 and AG Car. During this period $\dot{M}$ was also found to increase from $0.5-1.2 \times 10^{-4} M_{\odot} \mathrm{yr}^{-1}$, although it is still $\geq$ order of magnitude lower than required to form the dusty ejection nebula.

Comparison of both stellar and nebular properties of AFGL 2298 and AG Car show a very close correspondance between the two objects, the only differences being the present values of $\dot{M}$, and the possible presence of large dust grains around AG Car. We therefore suggest that both AFGL 2298 and AG Car are descendents of similar mass $\left(\geq 60 M_{\odot}\right)$ progenitors and are presently in the same evolutionary state. Further observations to better determine the stellar properties of AFGL 2298, their range and the timescale(s) of variability are therefore strongly urged.

Acknowledgements. This paper is partially based on observations obtained during service time with the United Kingdom Infrared Telescope - which is operated by the Joint Astronomy Centre on behalf of the U.K. Particle Physics and Astronomy Research Council we wish to thank the staff at UKIRT for their invaluable contribution to this work. We also thank Anna Pasquali for supplying her published spectra for inclusion in this work.

\section{References}

Appenzeller, I. 1986, in Luminous stars and associations in galaxies, ed. C. W. H. de Loore, A. Willis, \& P. Laskarides (Dordrecht: Reidel), IAU Symp., 116, 139
Becker, R. H., White, R. L., Helfand, D. J., \& Zoonematkermani, S. 1994, ApJS, 91, 347

Bohannan, B., \& Crowther, P. A. 1999, ApJ, 511, 374

Condon, J. J., Kaplan, D. L., \& Terzian, Y. 1999, ApJS, 123, 219

Crowther, P. A. 1997, in Luminous Blue Variables: Massive Stars in Transition, ed. A. Nota, \& H. Lamers, ASP Conf. Ser., 120, 51 Davidson, K. 1987, ApJ, 317, 760

Drissen, L., Crowther, P. A., Smith, L. J., et al. 2001, ApJ, 546, 484

Egan, M. P., Clark, J. S., Mizuno, D. R., et al. 2002, ApJ, 572, 288

Figer, D. F., Najarro, F., Morris, M., et al. 1998, ApJ, 506, 384

Figer, D. F., Morris, M., Geballe, T. R., et al. 1999, ApJ, 525, 759

Garcia-Lario, P., Manchado, A., Pych, W., \& Pottasch, S. R. 1997, A\&AS, 126, 479

Higgs, L. A., Wenker, H. J., \& Landecker, T. L. 1994, A\&A, 291, 295

Hillier, D. J., \& Miller, D. L. 1998, ApJ, 496, 407

Hillier, D. J., Crowther, P. A., Najarro, F., \& Fullerton, A. W. 1998, A\&A, 340, 483

Hillier, D. J., Davidson, K., Ishibashi, K., \& Gull, T. 2001, ApJ, 553, 837

Humphreys, R. M., \& Davidson, K. 1979, AJ, 232, 409

Lamers, H. J. G. L. M., Zickgraf F.-J., de Winter, D., Houziaux, L., \& Zorec, J. 1998, A\&A, 340, 117

Lamers, H. J. G. L. M., \& Nugis, T. 2002, A\&A, 395, L1

Leitherer, C., et al. 1994, ApJ, 428, 292

Morris, P. W., Eenens, P. R. J., Hanson, M. M., Conti, P. S., \& Blum, R. D. 1996, ApJ, 470, 597

Pasquali, A., \& Comeron, F. 2002, A\&A, 382, 1005 (PC02)

Stahl, O., Wolf, B., Klare, G., \& Juettner Cassatella, A. 1990, A\&A, 228,379

Ueta, T., Meixner, M., Dayal, A., et al. 2001, ApJ, 548, 1020 (U01)

Voors, R. H. M., Waters, L. B. F. M., de Koter, A., et al. 2000a, A\&A, 356, 501

Voors, R. H. M., Geballe, T. R., Waters, L. B. F. M., Najarro, F., \& Lamers, H. J. G. L. M. 2000b, A\&A, 362, 236

Wolf, B., Appenzeller, I., \& Stahl, O. 1981, A\&A, 103, 94 